\title{
ANÁLISE, REPROJETO E MELHORIA DO PROCESSO DE MONTAGEM DE EMBALAGENS DE MADEIRA UTILIZADAS PARA O ACONDICIONAMENTO DE MOTORES ELÉTRICOS
}

\section{ANALYSIS, REDESIGN AND IMPROVEMENT OF THE ASSEMBLY PROCESS OF WOODEN CONTAINERS USED FOR PACKAGING OF ELECTRIC MOTORS}

\author{
Luciano Costa Santos ${ }^{1}$; Cláudia Fabiana Gohr ${ }^{2}$; Marcos José Scharan ${ }^{3}$ \\ ${ }^{1}$ Universidade Federal da Grande Dourados - UFGD - Dourados/MS - Brasil \\ lucianosantos@ufgd.edu.br \\ ${ }^{2}$ Universidade Federal da Grande Dourados - UFGD - Dourados/MS - Brasil \\ claudiagohr@ufgd.edu.br \\ ${ }^{3}$ Universidade Federal de Santa Catarina - UFGD - Florianópolis/SC - Brasil \\ scharan@terra.com.br
}

\begin{abstract}
Resumo
Este artigo apresenta uma proposta de reestruturação do processo de montagem de caixas fechadas de madeira que são utilizadas como embalagem de motores elétricos e componentes relacionados. $O$ esforço de racionalização e padronização dos processos de montagem foi motivado pelo objetivo principal de aumentar a capacidade produtiva do sistema atual, que se encontrava ameaçado diante do crescimento de demanda previsto para os próximos anos. $O$ estudo tomou como base os itens mais representativos de quatro famílias de produtos, de modo a viabilizar a coleta de dados. Para desenvolver o projeto do novo sistema, foi necessário estabelecer uma sequência lógica dos fluxos, cronometrar e balancear os tempos produtivos, dimensionar a mão-de-obra necessária e reorganizar o layout industrial. A aplicação da metodologia proposta ocorreu segundo a abordagem da pesquisa-ação, na qual um dos autores desse artigo era o principal responsável pela intervenção organizacional para a melhoria dos processos. Os resultados demonstraram que o sistema proposto seria capaz de proporcionar um aumento da capacidade produtiva, com uma menor utilização de recursos que o sistema atual.
\end{abstract}

Palavras-chave: melhoria de processos; processos de montagem; projeto de fábrica.

\section{Introdução}

Tradicionalmente, a melhoria dos processos produtivos representa um dos problemas mais peculiares à atividade de um engenheiro de produção. A análise crítica e a padronização dos processos de manufatura foram impulsionadores da Engenharia Industrial no início do século XX, marcado pelos trabalhos de Taylor e Gilbreth com o surgimento dos estudos de tempos e movimentos. Atualmente, a abordagem de Taylor evoluiu ao incorporar princípios ergonômicos no 
projeto do trabalho e reconhecer que as organizações modernas dependem da interação dinâmica de seu sistema social com seu sistema técnico. Porém, mesmo com uma roupagem nova e mais adequada, a melhoria de eficiência nos sistemas produtivos continua sendo perseguida e acontece, via de regra, por meio da melhoria e da sistematização de processos.

No ambiente industrial, a melhoria de processos muitas vezes tem o objetivo de aumentar a produtividade e a capacidade de um sistema de produção. Frequentemente, essa melhoria implica em reorganizar fisicamente o sistema produtivo, demandando uma mudança do layout industrial para a otimização dos diferentes fluxos de processo. Para isso, é necessário conhecer os tempos produtivos e a sequência de etapas de cada um dos processos analisados.

Este artigo relata um típico trabalho de melhoria de processos em um sistema de produção de embalagens de madeira utilizadas para o acondicionamento de motores elétricos e componentes relacionados. A planta de montagem de embalagens é fornecedora interna de outras fábricas de um grande grupo empresarial que produz motores elétricos. Devido à expansão da empresa no exterior, a demanda por embalagens de madeira cresceu significativamente, com um destaque para um tipo específico de embalagem: as caixas fechadas. À medida que a demanda por estas caixas foi aumentando, o sistema atual de produção foi se adequando sem que houvesse um estudo detalhado do processo, gerando perdas, baixa produtividade e falta de capacidade.

Em uma análise preliminar, foi detectado que o processo atual de montagem de caixas fechadas teria dificuldades para atender à demanda prevista para os próximos anos. Essa constatação motivou a realização deste trabalho, uma vez que o sistema atual não possuía um fluxo de processo padronizado. Portanto, a execução deste trabalho foi norteada pelo objetivo de reestruturar e padronizar o processo de montagem de caixas fechadas para que o mesmo pudesse aumentar sua capacidade produtiva. Para isso, foi necessário estabelecer uma sequência lógica dos fluxos, cronometrar e balancear os tempos produtivos, dimensionar a mão-de-obra necessária e reorganizar o layout industrial.

A estrutura do texto apresentado inclui uma breve discussão teórica sobre os conceitos que deram base para a aplicação da metodologia adotada. Posteriormente, são relatados os procedimentos metodológicos que guiaram a coleta e a análise dos dados necessários para a realização do trabalho. Em seguida, é apresentada a análise do sistema atual e a construção de uma proposta que fosse capaz de resolver os problemas identificados. O texto é encerrado com uma comparação entre o sistema atual e o sistema proposto e com as considerações finais sobre o trabalho realizado.

\section{Base conceitual}


O desenvolvimento do trabalho se apoiou em conceitos essenciais para a área de gestão de produção: análise de processos, medição e balanceamento de tempos produtivos, layout industrial, produtividade e capacidade produtiva.

A análise dos processos produtivos parte do princípio que eles correspondem ao mecanismo de agregação de valor em um sistema de produção. Em um sentido amplo, os processos representam um conjunto de atividades logicamente inter-relacionadas que transformam entradas em saídas, agregando-lhes valor (PAIM et al., 2009, VERGIDIS; TURNER; TIWARI, 2008). De acordo com essa visão, é natural compreender a atividade de gestão da produção como uma atividade de gestão dos processos produtivos (SILVER, 2004). De fato, o foco em racionalização de processos, existente desde os primeiros trabalhos de Taylor e Gilbreth, continua sendo hoje uma temática de grande relevância em Engenharia de Produção e inclusive está presente em filosofias de gestão mais modernas, como manufatura enxuta e gestão da cadeia de suprimentos.

A melhoria de um processo depende diretamente da coleta e da análise de dados que são mensurados por meio de indicadores que a ajudam a estabelecer metas a serem alcançadas e servem de base para o seu redesenho (ADESOLA; BAINES, 2005). Dentre os indicadores de desempenho mais tradicionais dos processos produtivos, podem ser citados: tempo, capacidade e produtividade.

$\mathrm{O}$ estudo de tempos produtivos teve origem no início do século XX, quando Taylor resolveu cronometrar o trabalho de operários em uma indústria siderúrgica. Atualmente, a cronometragem industrial evoluiu bastante, tanto na forma que ela é realizada - incentivando o trabalhador a participar na definição de seus próprios padrões de trabalho (ADLER, 1993), quanto nos equipamentos e ferramentas de suporte - utilizando tecnologia digital e softwares sofisticados de análise (ELNEKAVE; GILAD, 2006). No entanto, a lógica na determinação do tempo padrão não mudou significativamente e ainda segue a sequência de passos que é usualmente difundida na literatura da área (NIEBEL; FREIVALDS, 2003, BARNES, 1977):

a) Dividir a operação em elementos de trabalho;

b) Cronometrar a operação na quantidade de vezes que for necessária para atender uma precisão e uma confiabilidade estabelecidas previamente;

c) Determinar o ritmo do operador observado, estabelecendo um coeficiente que deve ser incorporado ao tempo medido para normalizar as medições entre diferentes trabalhadores;

d) Determinar o fator de fadiga inerente à operação e as demais tolerâncias que a empresa se dispõe a conceder, incorporando esses fatores ao tempo normalizado para que se obtenha o tempo padrão.

O tempo padrão representa o tempo que um operador qualificado, trabalhando em um ritmo normal e usando ferramentas e procedimentos apropriados, irá gastar para completar uma tarefa (BAINES, 1995). O tempo padrão serve como parâmetro para a elaboração da maioria dos cálculos 
referentes ao processo produtivo e auxilia no estabelecimento de metas de melhoria para a operação.

A capacidade produtiva está vinculada ao tempo padrão e pode ser entendida como "o máximo nível de atividade de valor adicionado, em determinado período de tempo, que o processo pode realizar sob condições normais de operação" (SLACK et al., 1997, p. 346). A produtividade, por sua vez, é um indicador que relaciona a capacidade de produção com os recursos utilizados pelo processo (TANGEN, 2005). No nível dos processos produtivos, a produtividade é usualmente medida por tipo de recurso, geralmente mão-de-obra (peças/homem-hora) e máquina (peças/horamáquina), devido à maior facilidade de se obter esses dados no chão-de-fábrica.

Para aumentar a produtividade de um sistema de produção, pode ser necessário modificar o fluxo físico dos processos produtivos e, consequentemente, alterar o layout industrial. O layout industrial se preocupa com a disposição física dos recursos de transformação, isto é, máquinas, equipamentos e pessoas (MUTHER; WHEELER, 2008). Nesse sentido, o fluxo de materiais que permeia os recursos de transformação passa a ser um fator determinante para projetar ou reorganizar o layout de uma fábrica (TOMPKINS, 2002). Logo, se o redesenho dos processos produtivos modifica o fluxo dos mesmos, a alteração no fluxo pode demandar uma mudança na disposição física dos recursos de transformação.

A definição do fluxo do processo também determina o tipo de layout a ser projetado. De acordo com a natureza do processo, o arranjo físico da produção pode se enquadrar dentro de uma das quatro categorias básicas já consolidadas na literatura de gestão da produção (GAITHER; FRAZIER, 2001, SLACK et al., 1997): layout posicional, layout funcional, layout linear, layout celular.

Como nas linhas de produção os recursos são organizados de acordo com as etapas do processo de transformação, o problema fundamental dos layouts lineares e celulares é a distribuição de tarefas entre os diferentes postos de trabalho. Para isso, é necessário conhecer o tempo padrão de cada uma das operações, de forma que o equilíbrio entre os tempos produtivos possa garantir a estabilidade do processo. O procedimento de atribuir elementos de trabalho a um número mínimo de estações, respeitando as relações de precedências entre as tarefas, é normalmente denominado na literatura de "balanceamento de linha" (BOYSEN; FLIEDNER; SCHOLL, 2008).

No procedimento geral de balanceamento de linhas de montagem, as tarefas são distribuídas para as estações de trabalho após a determinação de seus tempos, de sua ordem de execução e do tempo de ciclo necessário para atender à demanda. Para a atribuição de tarefas existem diferentes métodos para a solução do problema, que normalmente dependem das diferentes variáveis envolvidas e dos objetivos do balanceamento (BOYSEN; FLIEDNER; SCHOLL, 2008, EREL; SARIN, 1998, GHOSH; GAGNON, 1989). 
Os problemas de balanceamento de linha são normalmente divididos em duas categorias: problema de balanceamento de linha de montagem simples (simple assembly line balancing problem - SALBP) e problema de balanceamento de linha de montagem generalizado (generalized assembly line balancing problem - GALBP). A diferença básica entre as duas categorias é que o GALBP está sujeito a restrições (ex.: restrições de espaço e tipos de atividades que podem ser combinadas), enquanto o SALBP é livre de restrições (GHOSH; GAGNON, 1989). Em geral, no balanceamento de linhas montagem mais curtas, ou mesmo de células de manufatura, o problema é abordado nas empresas na forma do SALBP, que é o caso do processo relatado neste artigo.

No entanto, seja qual for o método de balanceamento, um dos principais indicadores relacionados a esta tarefa é o tempo de ciclo. O tempo de ciclo (TC) corresponde ao tempo que transcorre entre as saídas de um processo. Esse indicador pode ser determinado de duas maneiras:

Pela relação entre o tempo disponível (TD) para a produção de um item e a capacidade $(\mathrm{C})$ do processo para produzir este item em um determinado período de tempo $(\mathrm{TC}=$ TD/C). Nesse caso, o tempo de ciclo é função da capacidade produtiva e determina o ritmo que pode ser alcançado pela linha de produção.

- Pela relação entre o tempo disponível (TD) para a produção de um item e sua demanda (D) em um determinado período de tempo (TC = TD/D). Nesse caso, o tempo de ciclo é função da demanda e determina o ritmo que é necessário para a linha de produção. Quando o tempo de ciclo é definido desse modo ele também pode ser chamado de takt time, uma nomenclatura característica do Sistema Toyota de Produção que evidencia a diferença conceitual entre as duas formas de cálculo (ALVAREZ; ANTUNES JR., 2001).

A revisão dos conceitos citados nesta seção foi fundamental para que pudessem ser definidos os procedimentos metodológicos que seriam adotados. Esses procedimentos são apresentados na seção a seguir.

\section{Procedimentos metodológicos}

A abordagem metodológica para a coleta e a análise de dados dos processos de montagem seguiu um roteiro detalhado com um conjunto de atividades que podem ser agrupadas em quatro etapas básicas, descritas a seguir.

Etapa 1: Identificação de famílias de produtos e seleção dos itens para análise. Os processos de montagem das caixas fechadas diferem conforme muda o formato de um item para outro. Como a empresa trabalha com um grande número de itens, foi definido que a análise não seria feita para todos os itens, caso contrário a coleta de dados seria inviabilizada. Portanto, a alternativa adotada foi dividir o conjunto total de itens em famílias de produtos que tinham 
processos em comum e, posteriormente, selecionar itens representativos de cada família para coletar os dados do sistema atual.

Etapa 2: Análise do sistema atual. Esta etapa foi composta pela análise do layout no qual as caixas são montadas, pela identificação da sequência de operações de cada processo e pela coleta de dados do sistema. Para a coleta de dados foi realizado um estudo de cronometragem que determinou o tempo padrão de cada uma das operações. Com os tempos padrões calculados, foi possível determinar outros indicadores relacionados, tais como, o tempo de ciclo, a capacidade produtiva, a produtividade de mão-de-obra e a quantidade de horas necessárias para atender à demanda.

Etapa 3: Projeto do novo sistema. O projeto do novo sistema de montagem foi iniciado com o redesenho do fluxo dos processos atuais. Para isso, foram montados postos de trabalho simulados, para que fosse possível testar diferentes alternativas de fluxo. Após a definição do fluxo preliminar para cada uma das famílias, foi feita a cronometragem das operações propostas e, em seguida, o balanceamento dos tempos produtivos, já que os processos seguiam um fluxo linear. Com o balanceamento, o número de operadores pôde ser dimensionado e as operações puderam ser reavaliadas, de modo a reunir as condições necessárias para a padronização dos processos.

Etapa 4: Avaliação de resultados. Após a determinação dos indicadores de processo (capacidade, produtividade, tempo de ciclo, etc.), foi possível comparar o sistema proposto com o sistema atual. Por meio dessa comparação, pôde-se identificar a evolução dos indicadores individuais e a evolução global do sistema. Por outro lado, a avaliação dos resultados teve o objetivo de fornecer parâmetros iniciais para o estabelecimento de metas para o processo de implantação das melhorias propostas.

Do ponto de vista da metodologia científica, a aplicação das etapas descritas acima seguiu a abordagem da pesquisa-ação. Na pesquisa-ação o pesquisador intervém na realidade organizacional e envolve o objeto pesquisado para o alcance dos objetivos da pesquisa (EDEN; HUXHAM, 1996, COUGHLAN; COGHLAN, 2002), o que naturalmente acontece quando os responsáveis pela melhoria de processos já fazem parte do ambiente pesquisado ou estabelecem uma equipe para a intervenção na empresa. Neste trabalho, um dos participantes da equipe era o principal responsável pela seção de embalagens e, consequentemente, pela intervenção organizacional para a melhoria dos processos.

Devido a um acordo de sigilo, foi decidido que o nome da empresa não seria divulgado neste artigo e todos os dados que pudessem identificar a organização seriam omitidos.

\section{Aplicação}


A aplicação da metodologia foi delimitada à seção de produção de embalagens da empresa, mais especificamente aos centros de trabalho destinados à montagem de caixas fechadas de madeira. É importante ressaltar que a seção de embalagens só executa processos de montagem, uma vez que os componentes de madeira são provenientes da serraria da empresa e vêm cortados nas dimensões exatas dos modelos de embalagens que são programados pelo departamento de PCP.

A seção de embalagens está dividida em sete centros de trabalho, cada um dedicado à montagem de famílias específicas, separadas de acordo com a semelhança em sua forma construtiva e, consequentemente, em seu processo de montagem. Dentre os sete centros de trabalho, dois são destinados à montagem de caixas fechadas, que representam o foco deste trabalho. Por questões de sigilo, estes centros de trabalho serão doravante denominados de CT1 e CT2.

\subsection{Identificação de famílias de produtos e seleção dos itens para análise}

Dentre os 471 itens de caixas fechadas que estão catalogados no sistema ERP da empresa, 451 são processados no CT1 e 20 itens são processados no CT2. Para cada um dos itens, foram analisadas as semelhanças segundo suas características de forma construtiva, tamanho e existência ou não de furações no fundo da caixa.

Para o agrupamento dos itens em famílias de produtos, foi utilizado o critério de similaridade entre as operações, que variavam de acordo com a forma construtiva de cada caixa. A partir desta análise foram formadas quatro famílias, que são descritas na figura 1 .

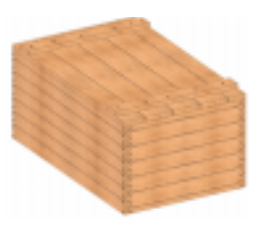

Família 1

Caixa KIT

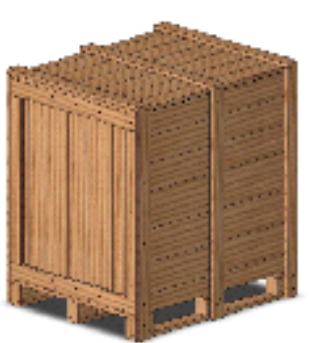

Família 2

Caixa CKD
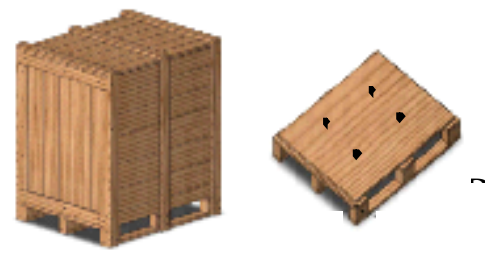

Família 3

Caixa CKD com furo

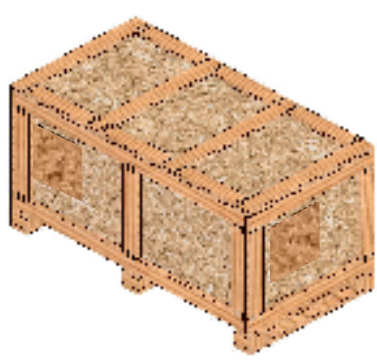

Família 4 Caixa OSB

Todas as famílias de caixas citadas na figura 1 são confeccionadas em madeira de pínus ou de eucalipto, tratadas termicamente e secas em estufa. A seguir, descrevem-se as características de cada família. 
Família 1 - Caixas para Kits: São caixas fechadas destinadas ao envio de peças de reposição para assistentes técnicos, clientes, representantes e filiais. Geralmente, acondicionam mais de uma peça de um mesmo item e sua capacidade de carga é limitada, sendo utilizada para acondicionar peças pequenas e mais leves.

Famílias 2 e 3 - Caixas CKD: São caixas fechadas destinadas ao envio de componentes de motores elétricos para as filiais no exterior. A sigla CKD provém do inglês (Complete Knock Down), termo utilizado para definir um produto completamente desmontado. Estas embalagens geralmente armazenam mais de um componente, pois são capazes de acondicionar peças de diferentes tamanhos e pesos em uma mesma caixa. Sua capacidade de carga pode chegar a $2000 \mathrm{~kg}$ e sua dimensão varia de acordo com o tamanho da peça. Na análise do processo de montagem, foi necessário desmembrar as caixas CKD em duas famílias, pois alguns itens possuíam uma operação a mais, relacionada com a forma construtiva que incluía furações na base da caixa. Portanto, as caixas que possuíam furações foram enquadradas dentro de outra família, denominada pela sigla CKDF.

Família 4 - Caixas OSB: São caixas específicas para o acondicionamento de inversores de frequência e soft starters. Possuem uma característica de utilizar madeira e chapas de OSB (Oriented Strand Board), um material constituído de fibras de madeira orientadas e coladas entre si formando chapas planas. Estas caixas possuem uma capacidade de carga limitada a $1000 \mathrm{~kg}$ e sua dimensão varia de acordo com o tamanho do produto. Outra característica destas caixas é que elas podem ser desmontáveis, pois seu fechamento é feito através de presilhas de aço.

As famílias que foram identificadas eram compostas de itens que passavam pelas mesmas operações e tinham a mesma forma construtiva. As únicas variações de um item para outro eram as dimensões de cada caixa e, em menor proporção, os tempos de montagem.

Para realizar a coleta de dados dos processos de montagem foi escolhido um item de cada família. Considerando que inicialmente não foram identificadas variações significativas nos tempos dos processos de uma mesma família, a análise de um único item foi uma opção que tornava o trabalho viável e ao mesmo tempo trazia uma perspectiva realista, embora fosse uma aproximação. A fim de obter uma maior representatividade do conjunto total de itens, foi escolhido o item de cada família que apresentava o maior volume de produção.

A escolha do item de maior volume foi confirmada pela análise da curva $\mathrm{ABC}$ de cada família, que demonstrou que os itens escolhidos pertenciam à classe A de suas famílias.

\subsection{Análise do sistema atual}


A seção de embalagens da empresa é localizada em um galpão próprio destinado à produção de diversos tipos de embalagens de madeira: engradados, paletes, bases, caixas fechadas, estrados e tabuleiros. Os centros de trabalho CT1 e CT2, que realizam a montagem de caixas fechadas, estão situados dentro da seção de embalagens, como mostra o layout esboçado na figura 2.

Figura 2 - Layout geral da seção atual de embalagens

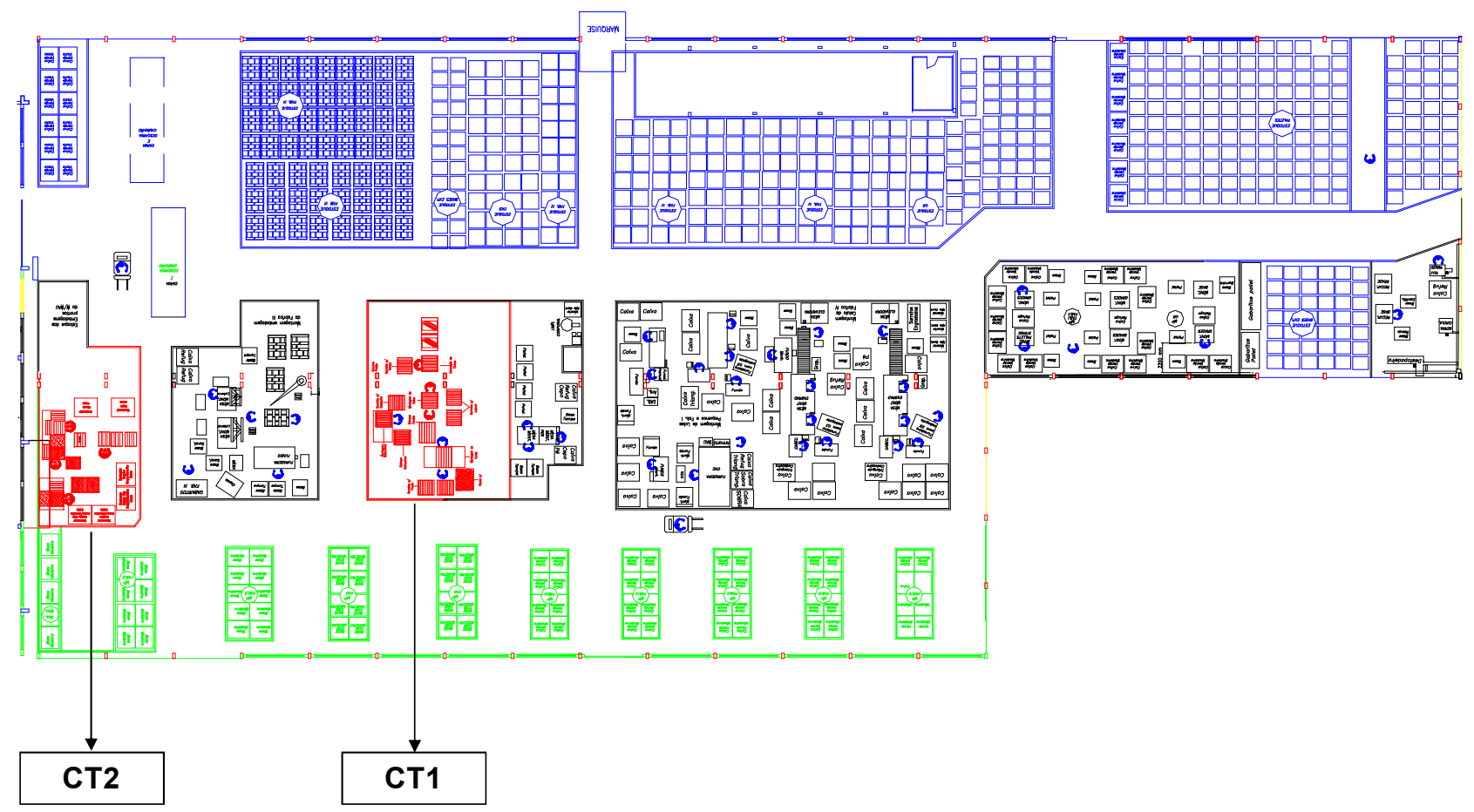

Atualmente, as caixas para Kits e CKD's são montadas exclusivamente no centro de trabalho CT1, enquanto as caixas OSB são montadas no centro de trabalho CT2. A figura 3 apresenta o layout dos dois centros de trabalho.

As caixas para Kits são montadas no início de cada turno de trabalho, considerando que atualmente são utilizados dois turnos de 8 horas e 48 minutos que, descontadas as horas destinadas a refeições e ginástica laboral, resultam em 8 horas efetivas de trabalho. A montagem das caixas dessa família é mais simples que as demais, sendo que os operadores executam individualmente a montagem completa de cada caixa.

Na rotina diária do $\mathrm{CT} 1$, as caixas CKD são montadas após a produção programada para as caixas de Kits em cada turno. A produção das caixas dessa família é realizada por três operadores em duas etapas. Como mostra a figura 3, a primeira etapa conta com três operadores (representados na cor azul), sendo que os operadores 1 e 2 montam todos os fundos de um determinado lote, formando um estoque intermediário de bases. Paralelamente, o operador 3 monta as respectivas cabeceiras. Na segunda etapa, os três operadores (representados na cor amarela) fazem a montagem da caixa na seguinte sequência: os operadores 1 e 2 unem o fundo e a cabeceira previamente 
montadas e fixam os sarrafos da lateral, em seguida, o operador 3 insere os sarrafos da tampa dentro da embalagem e as dispõe para serem carregadas para o estoque.

Figura 3 - Layout atual dos centros de trabalho CT1 e CT2
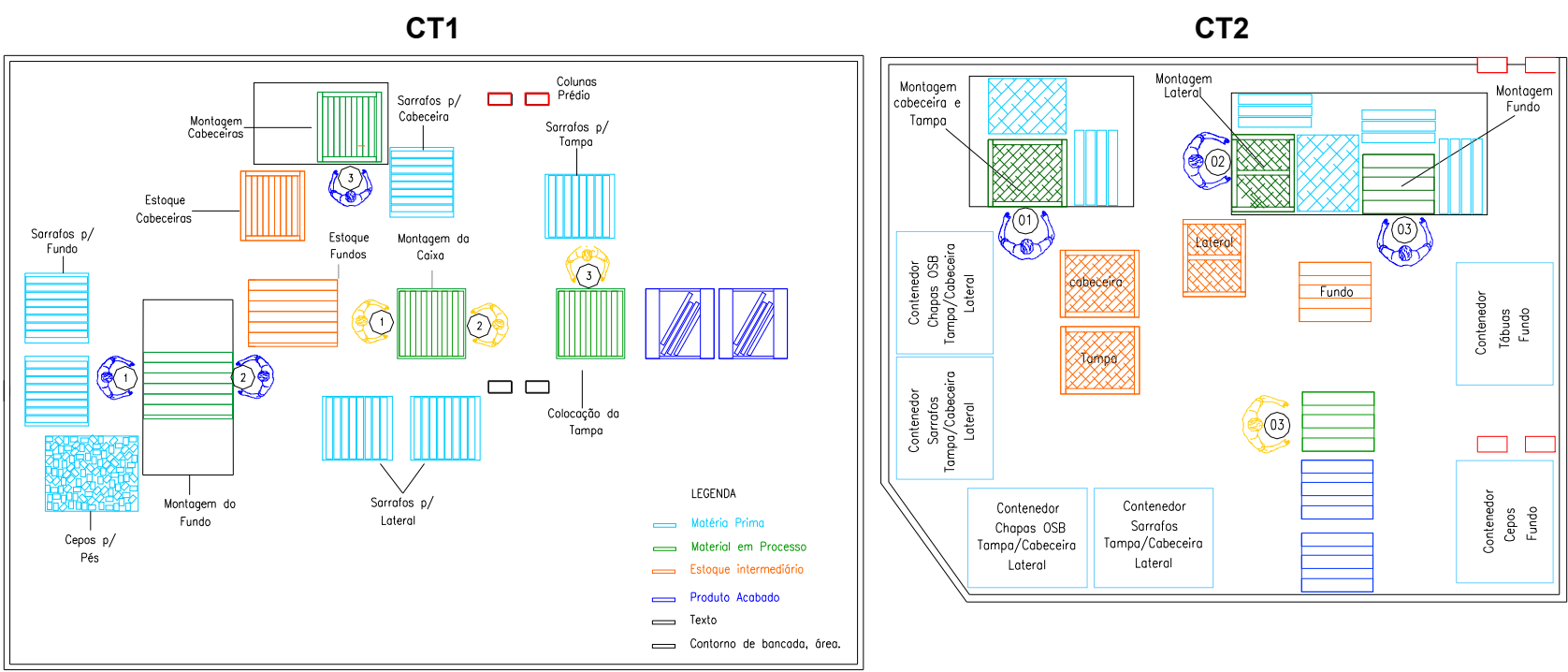

No centro de trabalho CT2, três operadores por turno realizam as atividades necessárias para a produção de caixas OSB. A sequência de montagem dessas caixas é a seguinte (como mostra a figura 3): o operador 1 monta as cabeceiras e a tampa e, paralelamente, o operador 2 monta as laterais e o operador 3 monta a base. Em seguida, o operador 3 forma kits agrupando uma base, duas cabeceiras, duas laterais e uma tampa, amarra e encaminha para o estoque, enquanto os operadores 1 e 2 vão realizar a montagem dos outros modelos de embalagens.

A coleta de dados para determinar os indicadores do sistema atual foi iniciada com a cronometragem das operações. Para isso, as operações foram divididas em elementos de trabalho, de forma a garantir uma maior precisão na cronometragem. Aos tempos medidos foram incorporados os fatores de ritmo dos operadores observados, o fator de fadiga inerente à operação e as demais tolerâncias utilizadas pela empresa. Com o estudo de tempos foi possível determinar o tempo padrão para a montagem dos itens selecionados de cada família.

A determinação do tempo de ciclo foi feita de acordo com a divisão de trabalho entre os operadores. O posto de trabalho que apresentava o maior tempo de produção representava o gargalo do processo. Dessa maneira, o tempo demandado pelo gargalo determinava o tempo de ciclo do processo. Somente na montagem de caixas para Kits o tempo padrão correspondeu ao tempo de ciclo, pois as etapas do processo de montagem não eram divididas em diferentes postos de trabalho, sendo que um único operador realizava a montagem completa de uma caixa. A tabela a seguir apresenta os dados obtidos no sistema atual. 
Tabela 1 - Dados quantitativos do sistema atual

\begin{tabular}{|c|c|c|c|c|c|c|c|c|c|}
\hline Família & $\begin{array}{c}\text { Centro } \\
\text { de } \\
\text { trabalho }\end{array}$ & $\begin{array}{c}\text { Tempo } \\
\text { padrão } \\
(\mathrm{min})\end{array}$ & $\begin{array}{l}\text { Tempo } \\
\text { de ciclo } \\
\text { (min) }\end{array}$ & $\begin{array}{l}\text { Capacidade } \\
\text { (peças/hora) }\end{array}$ & $\begin{array}{c}\mathrm{N}^{\circ} \text { de } \\
\text { operadores } \\
\text { por turno }\end{array}$ & Turnos & $\begin{array}{c}\text { Produtividade } \\
\text { (peças/ } \\
\text { homem·hora) }\end{array}$ & $\begin{array}{c}\text { Demanda } \\
\text { atual } \\
\text { (média diária) }\end{array}$ & $\begin{array}{c}\text { Horas } \\
\text { necessárias } \\
\text { (média) }\end{array}$ \\
\hline KIT & \multirow{3}{*}{ CT1 } & 1,81 & 1,81 & 99,36 & \multirow{3}{*}{3} & \multirow{4}{*}{2} & 33,12 & 112 & 1,13 \\
\hline CKD & & 11,05 & 5,75 & 10,43 & & & 3,48 & 79 & 7,57 \\
\hline CKDF & & 18,61 & 11,88 & 5,05 & & & 1,68 & 4 & 0,79 \\
\hline OSB & CT2 & 13,19 & 7,78 & 7,71 & 3 & & 2,57 & 40 & 5,19 \\
\hline
\end{tabular}

O número total de operadores foi determinado pela soma do número de funcionários de cada centro de trabalho nos dois turnos, resultando num total de doze operadores trabalhando na montagem das quatro famílias. Os dados de demanda foram originados do registro de vendas de cada item analisado. O cálculo da produtividade foi feito em relação à mão-de-obra, que é o recurso mais significativo para o processo de montagem de caixas fechadas, já que não são usadas máquinas complexas, mas somente ferramentas simples. Vale também destacar a última coluna da tabela 1, que apresenta a quantidade de horas necessárias para atender à demanda diária de cada item.

\subsection{Projeto do novo sistema}

Antes de projetar o novo sistema, os processos atuais foram analisados criticamente, a fim de estabelecer sequências de operações que pudessem ser padronizadas para cada uma das famílias de produtos. Essa análise adotou a premissa de que o novo sistema deveria proporcionar um aumento da capacidade produtiva, com a mínima utilização de recursos.

Com os novos fluxos definidos preliminarmente, foi feita a cronometragem das operações, que teve seus dados registrados em folhas como a apresentada na tabela 2.

Tabela 2 - Extrato de uma das folhas de cronometragem utilizadas

\begin{tabular}{|c|c|c|c|c|c|c|c|c|}
\hline \multicolumn{2}{|r|}{ Operação: } & \multicolumn{3}{|l|}{ Montar pé (caixas CKD) } & \multicolumn{4}{|c|}{ Obs.: somente 1 operador executa a operação. } \\
\hline \multicolumn{3}{|r|}{ Elementos } & $\begin{array}{l}\text { Tempo médio } \\
\text { cronometrado } \\
(\mathrm{min})\end{array}$ & $\begin{array}{c}\text { Ritmo } \\
(\%)\end{array}$ & $\begin{array}{c}\text { Tempo } \\
\text { normal } \\
(\mathrm{min})\end{array}$ & Frequência & $\begin{array}{c}\begin{array}{c}\text { Fator de } \\
\text { fadiga } \\
(\%)\end{array} \\
\end{array}$ & $\begin{array}{c}\text { Tempo } \\
\text { básico } \\
(\min )\end{array}$ \\
\hline 1 & \multicolumn{2}{|c|}{ Escolher 3 cepos e posicioná-los no gabarito de pé } & 0,100 & 100 & 0,100 & 3 & 11,00 & 0,334 \\
\hline 2 & \multicolumn{2}{|c|}{ Posicionar 1 sarrafo sobre os cepos } & 0,040 & 100 & 0,040 & 3 & 11,00 & 0,133 \\
\hline 3 & \multicolumn{2}{|c|}{ Pregar peças } & 0,068 & 100 & 0,068 & 3 & 11,00 & 0,227 \\
\hline 4 & \multicolumn{2}{|c|}{ Carimbar sarrafo do pé } & 0,195 & 100 & 0,195 & 3 & 8,00 & 0,632 \\
\hline 5 & \multicolumn{2}{|c|}{ Retirar do gabarito de pé e empilhar } & 0,070 & 100 & 0,070 & 3 & 11,00 & 0,234 \\
\hline & & & & & \multicolumn{3}{|r|}{ Total: } & 1,560 \\
\hline & & & & & \multicolumn{3}{|c|}{ Tempo padrão (tempo básico x 1,05): } & 1,638 \\
\hline
\end{tabular}

Após a determinação dos tempos padrões, foi realizado o balanceamento das operações de cada processo. A figura 4 apresenta um exemplo que contém os dados de balanceamento da montagem de caixas CKD. 
Figura 4 - Dados do processo de montagem proposto para as caixas CKD

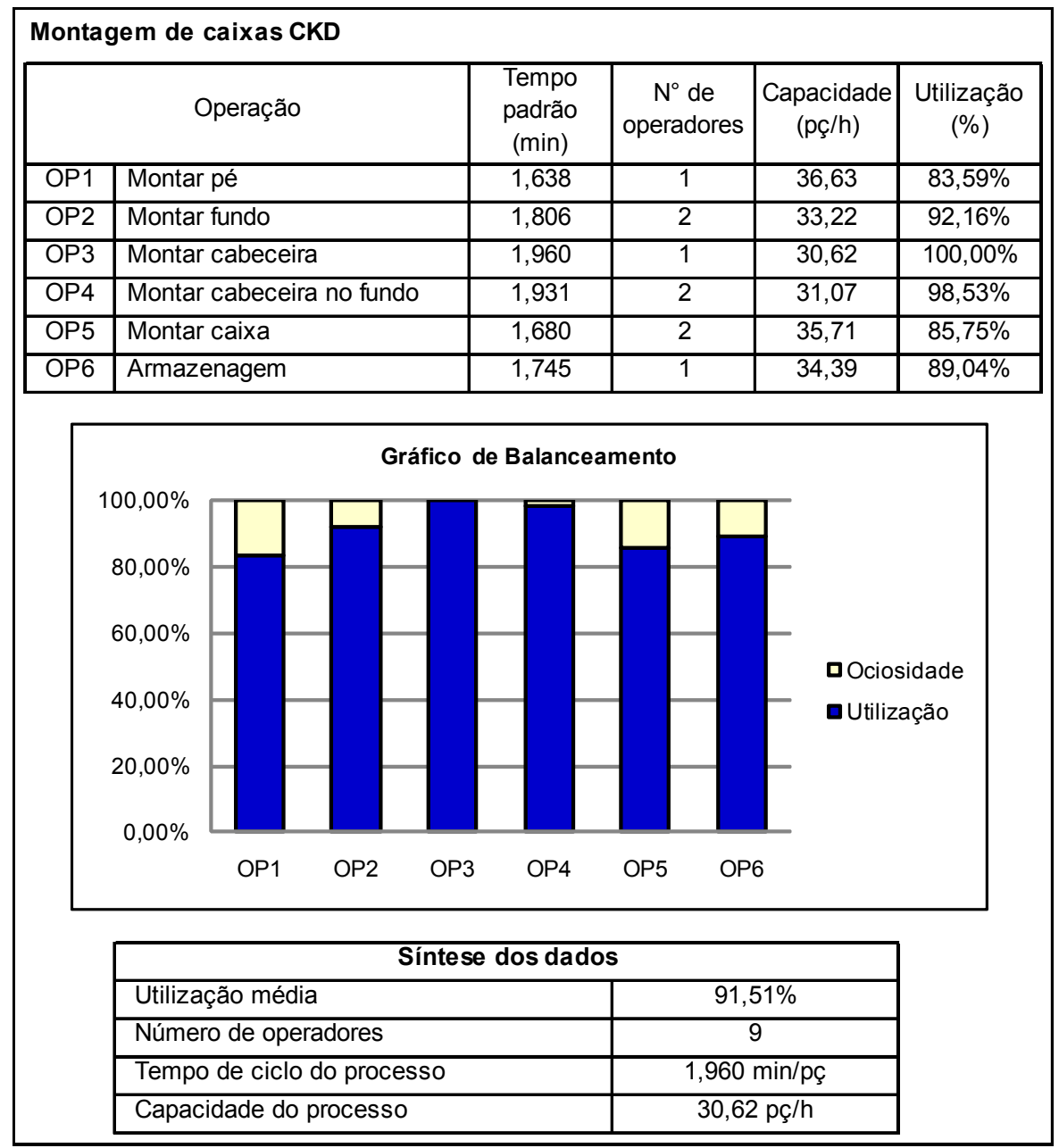

No projeto dos novos processos, as operações ocorrem de uma forma sequencial, tal como numa célula de produção, com operações simples e focalizadas em famílias de produtos. Considerando isso, o balanceamento foi feito de maneira que cada operação correspondesse a um posto de trabalho com um ou mais operadores.

Como se pode observar na figura 4, a determinação do tempo de ciclo dos processos ocorreu em função da capacidade produtiva, sendo que o tempo padrão da operação gargalo correspondia ao tempo de ciclo e limitava a capacidade do processo. Nesse caso, a operação gargalo adotava uma taxa de utilização de $100 \%$. Em função disso, as demais operações tinham uma taxa de utilização proporcional ao seu tempo padrão em relação ao tempo de ciclo do processo.

Também é interessante observar na figura 4 que a capacidade do processo foi registrada em valores com precisão de duas casas decimais. Isso foi feito para garantir uma maior confiabilidade nos cálculos, embora fosse admitido que os outputs de produção eram representados por números inteiros.

Na reorganização do layout foi definido que os processos de montagem das quatro famílias funcionariam em um único centro de trabalho. Com a correta sincronização da programação de 
montagem dos diferentes modelos, seria possível eliminar os desperdícios de recursos que ocorriam nos dois centros de trabalho atuais.

Como os diferentes processos de montagem não exigiam equipamentos específicos e de grande porte, foi possível organizar um layout com cinco bancadas de uso geral que seriam utilizadas totalmente ou parcialmente, de acordo com fluxo de processo de cada família. As bancadas foram dispostas de maneira a reduzir ao máximo a movimentação de materiais, respeitando os espaços necessários para cada posto de trabalho e conferindo a flexibilidade suficiente para comportar as quatro famílias de produtos.

Durante a montagem de cada família, o layout se comportaria como uma célula dedicada, que mudaria de configuração de acordo com a programação diária de montagem de caixas fechadas. As figuras 5, 6 e 7 apresentam o fluxo de montagem proposto para as famílias KIT, CKD, CKDF e OSB. É importante salientar que as famílias CKD e CKDF foram projetadas com o mesmo fluxo de montagem, variando somente nos tempos produtivos, já que a operação de furação foi incorporada na montagem da base das caixas CKDF.

Figura 5 - Fluxo do processo de montagem proposto para as caixas para Kits

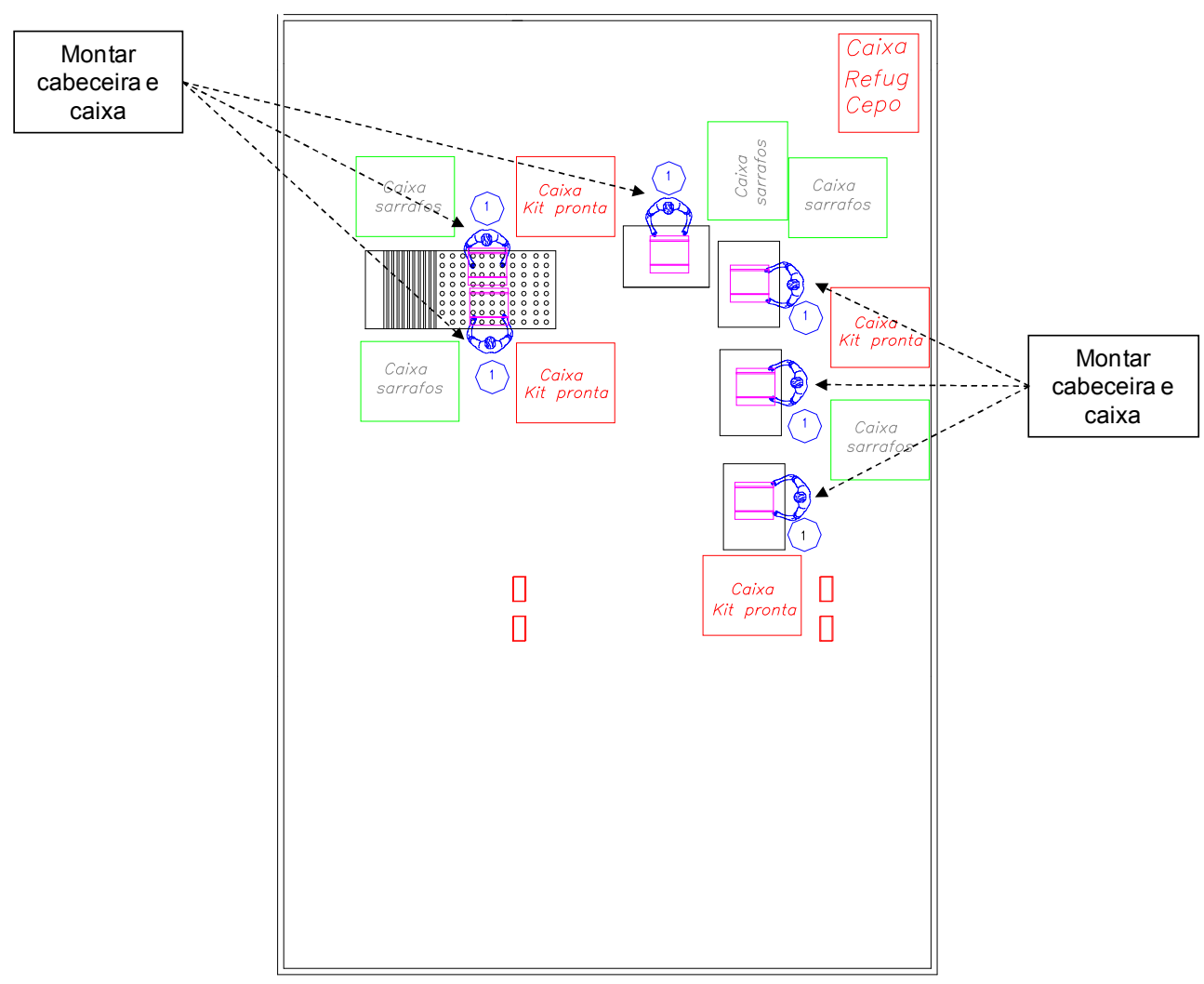


Figura 6 - Fluxo do processo de montagem proposto para as caixas CKD e CKDF

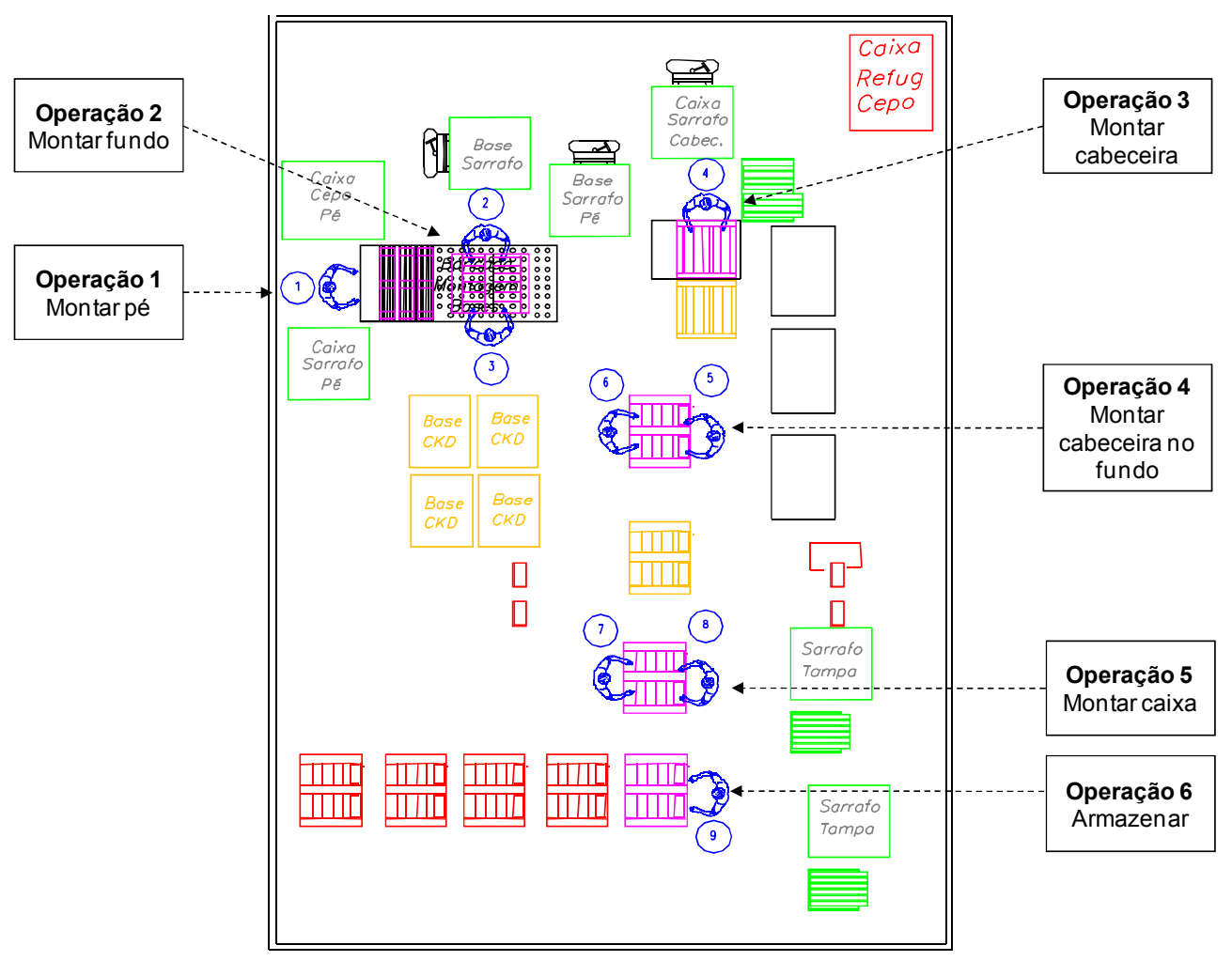

Figura 7 - Fluxo do processo de montagem proposto para as caixas OSB

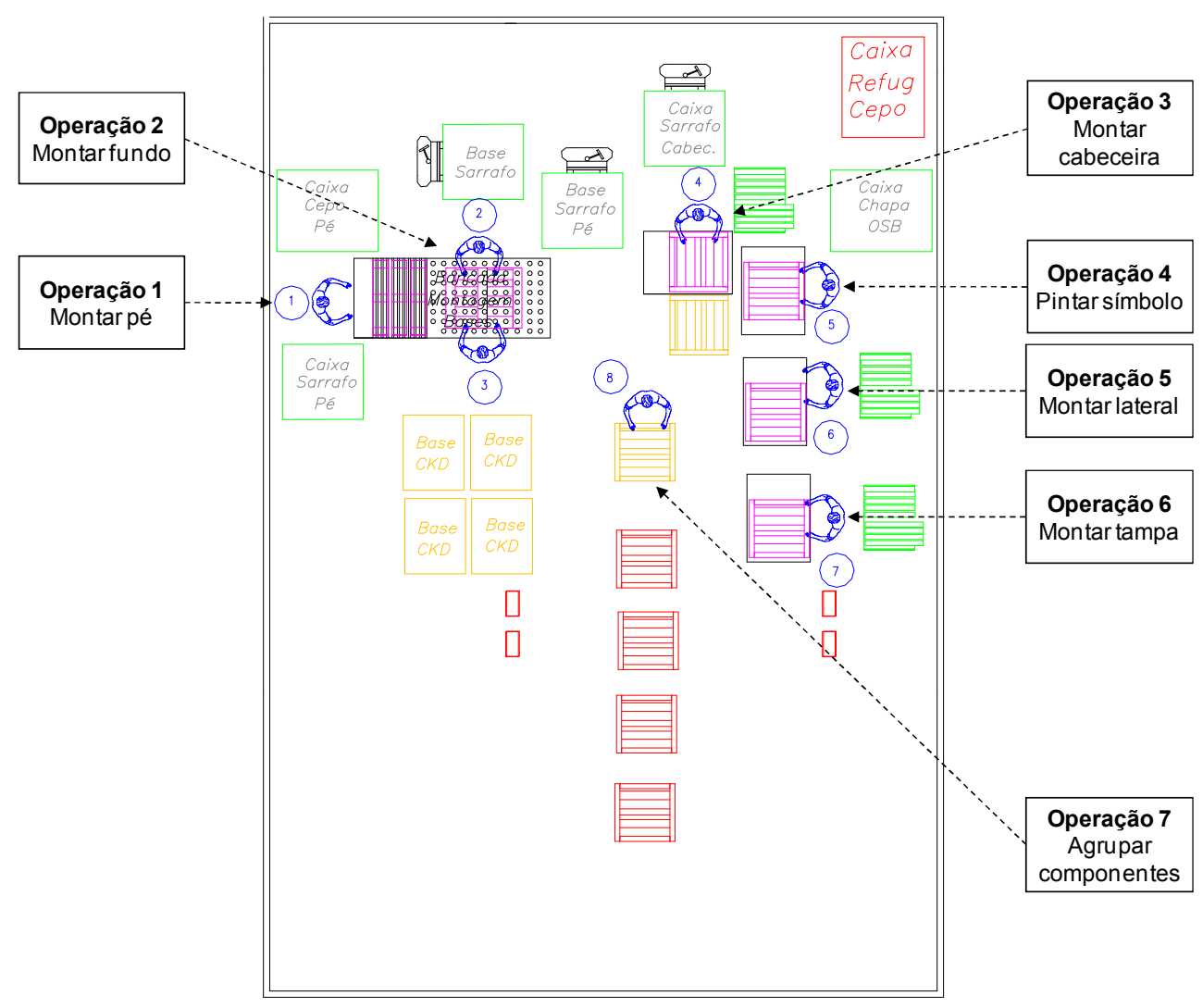


Convém ressaltar que no sistema proposto o fluxo de montagem das caixas para Kits não foi alterado, uma vez a equipe de análise de processos considerou que ele já estivesse adequado com os operadores executando individualmente a montagem completa de cada caixa. Porém, embora ele estivesse adequado, o número de operadores teria que ser dobrado para que fosse possível atender à demanda.

Como se trata de um projeto a ser implantado, o cálculo dos indicadores do sistema proposto tomou como base os valores previstos da demanda futura, já incluindo o crescimento que é esperado no curto prazo. A tabela a seguir apresenta os dados do sistema proposto.

Tabela 3 - Dados quantitativos do sistema proposto

\begin{tabular}{|c|c|c|c|c|c|c|c|c|c|}
\hline Família & $\begin{array}{c}\text { Centro } \\
\text { de } \\
\text { trabalho }\end{array}$ & $\begin{array}{c}\text { Tempo } \\
\text { padrão } \\
(\min )\end{array}$ & $\begin{array}{c}\text { Tempo } \\
\text { de ciclo } \\
(\mathrm{min})\end{array}$ & $\begin{array}{l}\text { Capacidade } \\
\text { (peças/hora) }\end{array}$ & $\begin{array}{c}\mathrm{N}^{\circ} \text { de } \\
\text { operadores } \\
\text { por turno }\end{array}$ & Turnos & $\begin{array}{c}\text { Produtividade } \\
\text { (peças/ } \\
\text { homem·hora) }\end{array}$ & $\begin{array}{c}\text { Demanda } \\
\text { prevista } \\
\text { (média diária) }\end{array}$ & $\begin{array}{c}\text { Horas } \\
\text { necessárias } \\
\text { (média) }\end{array}$ \\
\hline KIT & \multirow{4}{*}{ único } & 1,81 & 1,81 & 198,72 & 6 & \multirow{4}{*}{1} & 33,12 & 160 & 0,81 \\
\hline CKD & & 10,76 & 1,96 & 30,62 & \multirow{2}{*}{9} & & 3,40 & 100 & 3,27 \\
\hline $\mathrm{CKDF}$ & & 14,91 & 4,41 & 13,61 & & & 1,51 & 5 & 0,37 \\
\hline OSB & & 12,14 & 2,34 & 25,63 & 8 & & 3,20 & 70 & 2,73 \\
\hline
\end{tabular}

Com a análise dos dados do sistema, foi possível constatar que é possível atender à demanda trabalhando em um único turno, pois o somatório das horas necessárias para atender à demanda de cada família (adotando a premissa de que não existem variações significativas nos tempos produtivos de uma mesma família) era menor que a duração do turno de trabalho $(0,81+3,27+$ $0,37+2,73=7,18<8$ horas $)$

$\mathrm{O}$ número total de operadores foi determinado pelo dimensionamento da maior equipe dentre as quatro famílias, uma vez que todos os funcionários seriam alocados para o mesmo centro de trabalho em um único turno e estariam aptos para montar qualquer tipo de caixa fechada. Dessa maneira, a capacidade produtiva dos processos das famílias das caixas para Kits e das caixas OSB poderia ser ainda maior, já que esses processos teriam trabalhadores excedentes que poderiam auxiliar em atividades de apoio.

\subsection{Avaliação de resultados}

O sistema que foi proposto apresentou indicadores melhores que o sistema atual e ainda demonstrou capacidade produtiva para atender à demanda futura, com uma menor utilização de recursos. A tabela 4 e a figura 8 mostram uma comparação dos indicadores do sistema atual com os indicadores do sistema proposto. 
Tabela 4 - Comparação de resultados: sistema atual vs. sistema proposto

\begin{tabular}{|c|c|c|c|c|c|c|c|c|c|c|c|c|}
\hline \multirow{2}{*}{ Família } & \multicolumn{3}{|c|}{ Tempo padrão (min) } & \multicolumn{3}{|c|}{ Tempo de ciclo (min) } & \multicolumn{3}{|c|}{ Capacidade (peças/hora) } & \multicolumn{3}{|c|}{ Produtividade (peças/Hh) } \\
\hline & Atual & Proposto & Variação & Atual & Proposto & Variação & Atual & Proposto & Variação & Atual & Proposto & Variação \\
\hline KIT & 1,81 & 1,81 & - & 1,81 & 1,81 & - & 99,36 & 198,72 & $100,00 \%$ & 33,12 & 33,12 & - \\
\hline CKD & 11,05 & 10,76 & $-2,63 \%$ & 5,75 & 1,96 & $-65,92 \%$ & 10,43 & 30,62 & $193,43 \%$ & 3,48 & 3,40 & $-2,19 \%$ \\
\hline CKDF & 18,61 & 14,91 & $-19,90 \%$ & 11,88 & 4,41 & $-62,88 \%$ & 5,05 & 13,61 & $169,38 \%$ & 1,68 & 1,51 & $-10,21 \%$ \\
\hline OSB & 13,19 & 12,14 & $-7,96 \%$ & 7,78 & 2,34 & $-69,91 \%$ & 7,71 & 25,63 & $232,30 \%$ & 2,57 & 3,20 & $24,61 \%$ \\
\hline
\end{tabular}

Figura 8 - Gráficos comparativos: sistema atual vs. sistema proposto
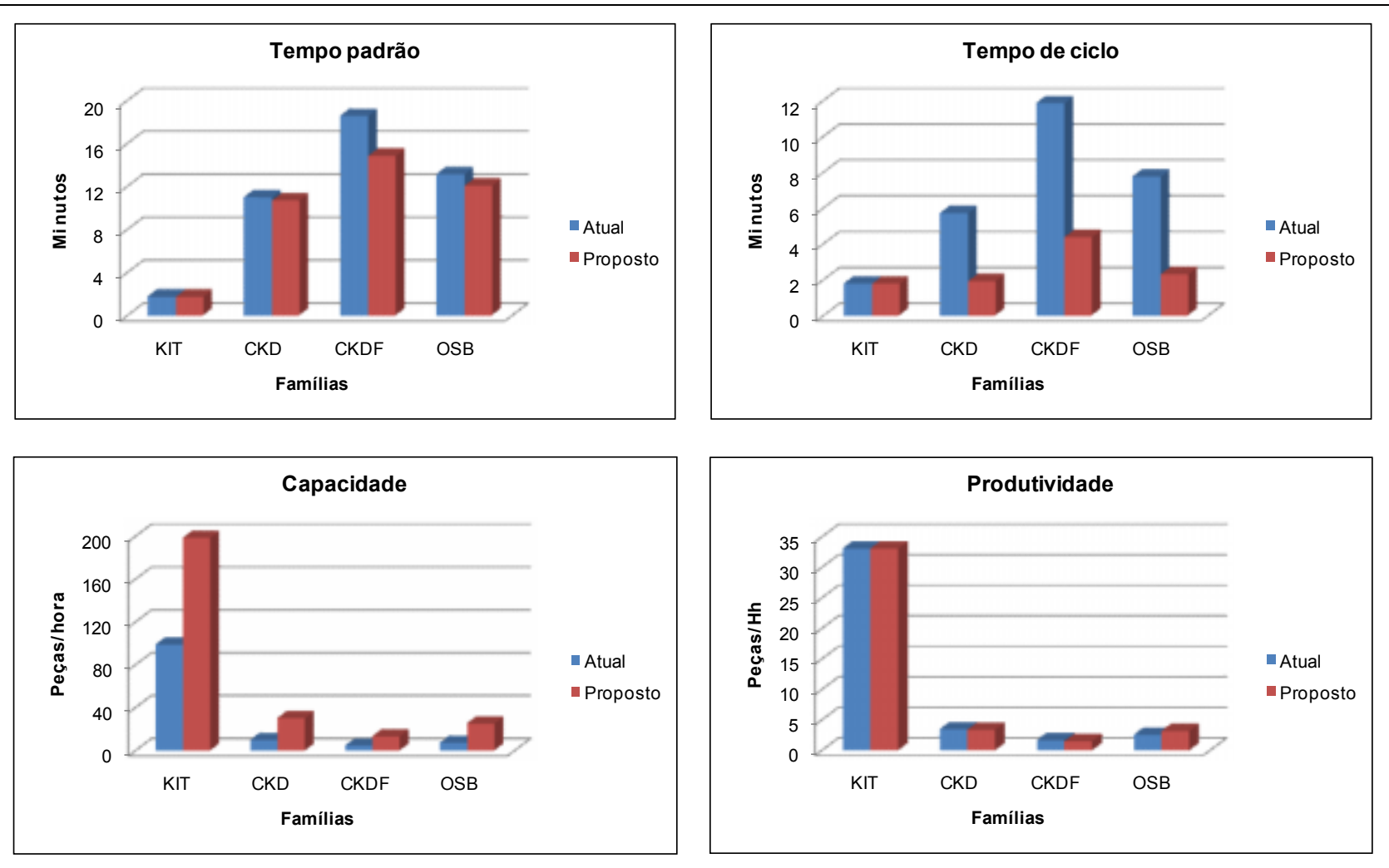

Como demonstram a tabela 4 e a figura 8 , os indicadores individuais de produtividade das caixas CKD e CKDF apresentaram um desempenho inferior no sistema proposto, embora tenha havido uma redução nos tempos produtivos e um aumento de capacidade. No entanto, quando se elaborou o cálculo da produtividade agregada, considerando a produção proporcional à demanda de cada família e totalizando a capacidade agregada de todas as famílias, o sistema proposto apresentou um desempenho muito superior. A tabela 5 demonstra a memória de cálculo da produtividade agregada (Atual $=52,26 / 6=8,71$; Proposto $=109,61 / 9=12,18$ ). Cabe destacar na tabela 5 que os recursos consumidos pelo processo são aqueles utilizados simultaneamente nos centros de trabalho. Por esse motivo, foram considerados 6 operadores no sistema atual (3 no CT1 e 3 no CT2) e 9 operadores no sistema proposto. 
Considerando as melhorias globais do sistema proposto, a tabela 6 apresenta uma síntese geral dos resultados alcançados.

Tabela 5 - Cálculo da produtividade agregada

\begin{tabular}{|c|c|c|c|c|c|c|c|c|c|c|c|c|}
\hline \multirow[t]{2}{*}{ Família } & \multicolumn{2}{|c|}{$\begin{array}{c}\text { Demanda } \\
\text { (média diária) }\end{array}$} & \multicolumn{2}{|c|}{$\begin{array}{c}\text { Demanda } \\
\text { proporcional } \\
\text { (média diária) }\end{array}$} & \multicolumn{2}{|c|}{$\begin{array}{l}\text { Capacidade } \\
\text { (peças/hora) }\end{array}$} & \multicolumn{2}{|c|}{$\begin{array}{c}\text { Produção } \\
\text { proporcional } \\
\text { (peças/hora) }\end{array}$} & \multicolumn{2}{|c|}{$\begin{array}{c}\text { Recursos } \\
\text { utilizados por } \\
\text { hora (operadores) }\end{array}$} & \multicolumn{2}{|c|}{$\begin{array}{c}\text { Produtividade } \\
\text { agregada } \\
\text { (peças/Hh) }\end{array}$} \\
\hline & Atual & Prevista & Atual & Prevista & Atual & Proposto & Atual & Proposto & Atual & Proposto & Atual & Proposto \\
\hline KIT & 112 & 160 & $47,66 \%$ & $47,76 \%$ & 99,36 & 198,72 & 47,36 & 94,91 & \multirow{5}{*}{6} & \multirow{5}{*}{9} & \multirow{5}{*}{8,71} & \multirow{5}{*}{12,18} \\
\hline CKD & 79 & 100 & $33,62 \%$ & $29,85 \%$ & 10,43 & 30,62 & 3,51 & 9,14 & & & & \\
\hline CKDF & 4 & 5 & $1,70 \%$ & $1,49 \%$ & 5,05 & 13,61 & 0,09 & 0,20 & & & & \\
\hline OSB & 40 & 70 & $17,02 \%$ & $20,90 \%$ & 7,71 & 25,63 & 1,31 & 5,36 & & & & \\
\hline Total & 235 & 335 & $100,00 \%$ & $100,00 \%$ & - & - & 52,26 & 109,61 & & & & \\
\hline
\end{tabular}

Tabela 6 - Síntese da comparação de resultados

\begin{tabular}{|l|c|c|c|}
\hline Indicadores & Atual & Proposto & Ganho \\
\hline Número de operadores & 12 & 9 & $25,00 \%$ \\
\hline Área ocupada $\left(\mathrm{m}^{2}\right)$ & 206 & 167 & $18,93 \%$ \\
\hline Horas necessárias para atender à demanda & 14,68 & 7,18 & $51,09 \%$ \\
\hline Turnos de trabalho & 2 & 1 & $50,00 \%$ \\
\hline Capacidade agregada (peças/hora) & 52,26 & 109,61 & $109,74 \%$ \\
\hline Produtividade agregada (peças/homem-hora) & 8,71 & 12,18 & $39,84 \%$ \\
\hline
\end{tabular}

A comparação entre os indicadores do sistema atual com os indicadores do sistema proposto encorajou a empresa a implantar as modificações sugeridas pela equipe de melhoria. Após a implantação do sistema proposto, a análise deverá ser realizada novamente para possíveis ajustes.

\section{Considerações finais}

A lógica da racionalização industrial foi discutida neste artigo, que demonstrou como pequenas modificações no fluxo dos processos podem trazer grandes benefícios no desempenho da produção. Muitas vezes, processos simples e pouco desenvolvidos tecnologicamente, tais como os processos de montagem de embalagens de madeira, são negligenciados na priorização dos esforços de melhoria. Entretanto, muitos dos processos de baixa complexidade são intensivos em mão-deobra e inerentemente ineficientes, o que revela o grande potencial de melhoria e padronização de operações.

A reestruturação do fluxo dos processos de montagem de caixas fechadas incluiu a modificação do projeto do layout dos centros de trabalho atuais. Essas mudanças, associadas à reorganização das operações e à realocação dos postos de trabalho, provaram que o sistema proposto conseguirá atingir níveis de capacidade compatíveis com o crescimento da demanda 
futura. Como reforço a essa constatação, a melhoria dos índices relacionados com a eficiência produtiva representa um incentivo para a implantação do sistema proposto.

Em relação aos indicadores do processo, cabe destacar o cálculo da produtividade agregada, demonstrado na tabela 5. A utilização desse indicador chamou a atenção para o cuidado que se deve ter ao analisar indicadores individuais em detrimento da visão global do processo. No caso relatado neste artigo, a produtividade individual dos processos de montagem de duas famílias havia caído na projeção feita para o sistema proposto. Porém, quando se comparou a produtividade agregada dos dois sistemas (atual vs. proposto), foi possível identificar a melhoria global nesse indicador.

O procedimento de quatro passos para análise e reprojeto do sistema produtivo provou sua aplicabilidade na montagem caixas fechadas, guiando todo o trabalho de campo para a melhoria do processo. Por esse motivo, a estrutura de desenvolvimento desse trabalho poderá ser utilizada sem maiores dificuldades em outros esforços semelhantes a serem realizados na empresa.

Entretanto, a aplicação dessa metodologia merece considerações adicionais. Na aplicação que foi apresentada aqui, pode-se advertir que o sistema proposto ainda deve ser ajustado no momento da implantação ou com estudos adicionais que dão continuidade a este trabalho. Como foi escolhido apenas um item de cada família para a análise, torna-se importante estudar os efeitos provenientes da produção simultânea do mix total de produtos. A análise de um único item de cada família também impossibilitou o estudo dos tempos entre trocas de lotes, assunto que necessariamente deverá ser objeto de investigação em futuros trabalhos.

\begin{abstract}
This paper presents a proposal for restructuring the process of assembling closed wooden boxes that are used for packaging of electric motors and related components. The effort to improve and standardize the assembly processes was motivated by the main purpose of increasing the productive capacity of the current system, which was threatened by the anticipated growth in demand for years to come. The study was based on the items most representative of four product families in order to facilitate data collection. To develop the design of the new system was necessary to establish a logical flow, measure and balance the productive time, calculate the manpower required and restructure the industrial layout. The proposed methodology was applied according to the approach of action research, in which one of the authors of this paper was primarily responsible for organizational intervention to improve processes. The results demonstrated that the proposed system would be able to provide an increase in production capacity, with less use of resources that the current system.
\end{abstract}

Key-words: process improvement; assembly processes; factory design.

\title{
Referências
}

ADESOLA, S.; BAINES, T. Developing and evaluating a methodology for business process improvement. Business Process Management Journal, v. 11, n. 1, p. 37-46, 2005.

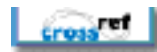

ADLER, P. S. Time-and-motion regained. Harvard Business Review, v. 71, n. 1, p. 97-108, 1993.

ALVAREZ, R. R.; ANTUNES JR., J. A. V. Takt-time: conceitos e contextualização dentro do Sistema Toyota de Produção. Gestão \& Produção, v. 8, n. 1, p. 1-18, 2001. 
BAINES, A. Work measurement - the basic principles revisited. Work Study, v. 44, n. 7, p. 10-14, 1995.

Lresiset

BARNES, R. M. Estudo de movimentos e de tempos: projeto e medida do trabalho. São Paulo: Edgard Blücher, 1977.

BOYSEN, N.; FLIEDNER, M.; SCHOLL, A. Assembly line balancing: which model to use when? International Journal of Production Economics, v. 111, n. 2, p. 509-528, 2008.

crosiset

COUGHLAN, P.; COGHLAN, D. Action research for operations management. International Journal of Operations \& Production Management, v.22, n.2, p.220-240, 2002.

creas ret

EDEN, C.; HUXHAM, C. Action research for management research. British Journal of Management, v. 7, n. 1, p. $75-86,1996$.

crosiset

ELNEKAVE, M.; GILAD, I. Rapid video-based analysis system for advanced work measurement. International Journal of Production Research, v. 44, n. 2, p. 271-290, 2006.

creas ret

EREL, E.; SARIN, S. C. A survey of the assembly line balancing procedures. Production Planning \& Control, v. 9, n. 5, p. 414-434, 1998.

crosiset

GAITHER, N.; FRAZIER, G. Administração da produção e operações. 8.ed. São Paulo: Pioneira Thomson Learning, 2001.

GHOSH, S.; GAGNON, R. J. A comprehensive literature review and analysis of the design, balancing and scheduling of assembly systems. International Journal of Production Research, v. 27, n. 4, p. 637-670, 1989.

cresiset

MUTHER, R.; WHEELER, J. D. Planejamento simplificado de layout: sistema SLP. São Paulo: IMAM, 2008.

NIEBEL, B. W.; FREIVALDS, A. Methods, standards and work design. 11.ed. New York: McGraw-Hill Irwin, 2003.

PAIM, R. et al. Gestão de processos: pensar, agir e aprender. Porto Alegre: Bookman, 2009.

SILVER, E. A. Process management instead of operations management. Manufacturing \& Service Operations Management, v. 6, n. 4, p. 273-279, 2004.

areaset

SLACK, N. et al. Administração da produção. São Paulo: Atlas, 1997.

TANGEN, S. Demystifying productivity and performance. International Journal of Productivity and Performance Management, v. 54, n. 1, p. 34-46, 2005.

crosiset

TOMPKINS, J. A. et al. Facilities planning. 3.ed. New York: John Wiley \& Sons, 2002.

VERGIDIS, K.; TURNER, C. J.; TIWARI, A. Business process perspectives: theoretical developments vs. real-world practice. International Journal of Production Economics, v. 114, n.1, p. 91-104, 2008.

trosiset 


\section{Dados dos autores:}

Nome completo: Luciano Costa Santos

Filiação institucional: Universidade Federal da Grande Dourados

Departamento: Faculdade de Engenharia (não há departamento)

Função ou cargo ocupado: Professor Adjunto

Endereço completo para correspondência (bairro, cidade, estado, país e CEP):

Rua Major Capilé, 1135, apto. 1004

Jardim Central

Dourados - MS, Brasil

CEP: 79.805-010

Telefones para contato:

(67)3410-2174

(67)8142-2184

e-mail:1ucianosantos@ufgd.edu.br

Nome completo: Cláudia Fabiana Gohr

Filiação institucional: Universidade Federal da Grande Dourados

Departamento: Faculdade de Administração, Ciências Contábeis e Economia (não há departamento)

Função ou cargo ocupado: Professora Adjunta

Endereço completo para correspondência (bairro, cidade, estado, país e CEP):

Rua Major Capilé, 1135, apto. 1004

Jardim Central

Dourados - MS, Brasil

CEP: $79.805-010$

Telefones para contato:

(67)3410-2051

(67)8143-9940

e-mail: claudiagohr@ufgd.edu.br

Nome completo: Marcos José Scharan

Filiação institucional: Universidade Federal de Santa Catarina

Departamento: Engenharia de Produção e Sistemas

Função ou cargo ocupado: Estudante de Pós-Graduação

Endereço completo para correspondência (bairro, cidade, estado, país e CEP): 
Av. Pref. Waldemar Grubba, $n^{\text {o: }}: 3000$

Vila Lalau

Jaraguá do Sul - SC, Brasil

CEP: 89.256-900

Telefones para contato:

(47) $3276-4610$

(47) 9616-9129

e-mail:scharan@terra.com.br

Recebido para publicação em: 20/10/2010

Aceito para publicação em: 01/12/2010 